\title{
A Survey of Personnel Protective equipment's (PPE) Use and Comfort Levels Among Surgeons During Routine Cancer Surgery in the COVID-19 Pandemic
}

\author{
Shivakumar Thiagarajan ${ }^{1}$ (D) Preethi Shetty ${ }^{1} \cdot$ Aashish Gulia $^{1} \cdot$ Gagan Prakash ${ }^{1} \cdot$ C. S. Pramesh ${ }^{1} \cdot$ Ajay Puri $^{1}$
}

Received: 18 January 2021 / Accepted: 21 March 2021 / Published online: 26 March 2021

(C) Indian Association of Surgical Oncology 2021

\begin{abstract}
Personnel protective equipment (PPE) are recommended during surgery even in COVID-19 negative patients especially in a highprevalence region due to its higher false-negative rates. However, the use of PPE has not been universal mainly due to the perception of discomfort and associated stress and fatigue. This study was done to understand the pattern of PPE use by cancer surgeons during the pandemic and the associated discomfort, stress, and fatigue with its use. The survey, consisting of 29 questions, was circulated widely across the country by email and chat groups among cancer surgeons. The study was registered with the Clinical Trials Registry of India (CTRI/2020/08/027050). We received a total of 342 evaluable responses that could be included for analysis. All the respondents used appropriate PPE in different combinations. N-95 mask and the face shield were the two components of the PPE that gave rise to a lot of discomforts. Fogging (of face shields) $(p<0.001$, OR 3.61), dryness of mouth $(p<0.001$,OR 3.35), and breathing difficulty/feeling of suffocation $(p=0.034$, OR 1.68) contributed to the stress, whereas headache $(p<0.001$,OR 11.34$)$ and breathing difficulty/feeling of suffocation $(p<0.001$,OR 4.24) contributed to the fatigue associated with PPE use the most. PPE was routinely used during cancer surgery in COVID-19 negative patients during the pandemic. However, most surgeons experienced different degrees of discomfort, especially with the N-95 masks and eye protection.
\end{abstract}

Keywords PPE $\cdot$ COVID-19 negative $\cdot$ Routine $\cdot$ Cancer surgery $\cdot$ Discomfort

\section{Introduction}

The risk to the $\mathrm{HCW}$ in the operating room (OR) is high due to close contact with the patient's body fluids, especially during aerosol-generating procedures and in longer duration surgeries [1]. The regular use of personnel protective equipment (PPE) has been recommended in all surgical procedures [2]. It is important to don appropriate personal protective equipment (PPE) during surgery even in COVID-19 negative patients tested with RT-PCR due to the high false-negative rate, especially when the prevalence rate in that geographical

Shivakumar Thiagarajan drshiva78in@gmail.com

1 Department of Surgical Oncology, Tata Memorial Hospital, Homi Bhabha National Institute (HBNI), Parel, Mumbai, India region is high [3]. The usage of PPE has proven to be successful in preventing and limiting the spread of the infection previously $[4,5]$. However, there is associated discomfort with prolonged duration usage. The regular use of PPE has resulted in various health-related issues (headaches, dehydration, and contact dermatitis) [6, 7]. Studies have shown that when HCW continue to work for $>1 \mathrm{~h}$ with $\mathrm{N}-95$ masks without a break, the blood $\mathrm{CO}_{2}$ levels may increase causing various physiological effects such as headache and increased work of breathing among others [8].

During an audit of the COVID-19 briefing and debriefing form at our institute (a tertiary cancer center), it was noted that the compliance to PPE while operating on COVID-19negative patients was good, but not $100 \%$ [9]. The subsequent survey done highlighted certain problems associated with PPEs use during routine surgeries. Face shields were among the components that were not routinely used, especially by the surgeons, due to poor visibility, fogging, and discomfort [9]. Existing literature suggests that the use of the various PPEs 
leads to surgeon discomfort and contributes to surgeon fatigue as well [10]. We undertook this nationwide survey among surgical oncologists to assess the pattern of PPE use in routine cancer surgery in COVID-19-negative patients during the pandemic and the prevalence and magnitude of discomfort and/or fatigue attributable to its use.

\section{Methods}

We developed a survey that consisted of 29 questions (Appendix 1) after a detailed literature review and deliberations regarding the various issues faced with the use of PPE during surgery in COVID-19-negative patients since the onset of the pandemic. The questions were also tailored based on our previous survey done [9]. The survey was divided into three sections, the first section had questions related to the demographic details of the surgeons, including the number of surgeries performed during the nationwide lockdown; the second section had questions related to the various personal protective equipment (PPE) used during these surgeries; the third section had questions assessing the discomfort and fatigue related to the use of PPE along with mechanisms adopted to cope with them. The study was approved by our institutional ethics committee and pre-registered with the Clinical Trials Registry of India (CTRI/2020/08/027050) prior to the start of the study.

An electronic questionnaire-based survey was created using the SurveyMonkey platform (Survey Monkey Inc., San Mateo, CA). The link (https://www.surveymonkey.com/ r/8LM7JDW) for this web-based cross-sectional survey with an introductory message explaining the purpose of the survey was widely circulated among surgical oncologists practicing in different parts of India via email/chat messages through personal contacts, mailing lists, and chat groups of surgical societies and institutional alumni. The anticipated time to complete the survey was $8 \mathrm{~min}$. The survey link was kept open for 4 weeks between 11 August 2020 and 8 September 2020 . Three reminders were sent at regular intervals (10 days) including one before the closure of the survey.

Statistical analysis was done with SPSS version 24 (IBM Corp, Armonk, New York). Quantitative analysis of the responses to the survey questions was done to understand the use of PPE and the associated discomfort and fatigue. The chisquare test was done to understand the stress, fatigue, and inability to enjoy surgery caused by various components of the PPE (univariate analysis). Binary logistic regression (multivariate analysis) was subsequently performed to identify the various factors associated with the different PPE components that maximally contributed to stress, fatigue, and inability to enjoy surgery. A $p$ value of 0.05 was considered statistically significant. No adjustments were made for multiple comparisons.

\section{Results}

A total of 374 responses were received from the nationwide survey. Most of those who started the survey completed it $(88 \%)$, and those who completed it took an average time of $6 \mathrm{~min} 25 \mathrm{~s}$. Out of the 374 responses, 32 had to be excluded (Fig. 1), and the remaining 342 responses were included for the final analysis.

\section{Demography of the Respondents (Table 1)}

The majority of our respondents had a dedicated site-specific oncology practice (operated only on certain sites, e.g., head and neck, breast, etc.) $(n=241,70.4 \%)$ (Fig. 2). There was fairly even distribution among the respondents between private $(n=176,51.5 \%)$ and publicly funded hospitals $(n=155$, $45.3 \%$ ) (Table 1). The approximate number of cancer surgeries performed in these hospitals during the pandemic was $\leq 10$ cases/day $(n=298,87.1 \%)$. Almost all publicly funded hospitals (except two) performed routine preoperative tests for COVID-19. In the private sector, 21 out of the 176 centers did not perform the routine preoperative test for COVID-19 $(p<0.001)$.

\section{Pattern of PPE Usage (Table 2)}

N-95mask/eye protection devices/double gloves/reusable gowns were the most common combination of PPE used by surgeons in publicly funded hospitals, whereas the N-95 masks/eye protection devices/double gloves/disposable gowns/shoe covers were the most common combination used by surgeons in the private sector. A large majority of the surgeons $(n=280,81.8 \%)$ were in agreement with the PPE policy adopted by their respective institutes/centers. N-95 with a 3-ply mask was the most commonly used mask combination ( $n=115,33.6 \%)$, and face shields were often used for eye protection $(n=180,52.6 \%)$. Most surgeons used eye protection during routine surgeries $(n=264,77.2 \%)$, but a few surgeons used them only in surgeries that involved aerosolgenerating procedures $(n=61,17.8 \%)$.

\section{Discomfort and Fatigue Attributable to the Usage of PPE (Table 2)}

The discomfort and fatigue associated with the routine use of PPE are summarized in Table 3. Most surgeons experienced varying degrees of discomfort though they were able to continue operating despite the discomfort. Most surgeries lasted for $3 \mathrm{~h}$ or more $(n=203,59.3 \%)$. Nearly one-fourth of the surgeons ( $n=82,24 \%$ ) had to take a break in between surgery due to the discomfort caused by the use of PPE. Face masks gave rise to a variety of issues such as difficulty in communication, dryness of mouth, breathing difficulties/ suffocation, 
Fig. 1 Flowchart giving details of the respondents included and excluded

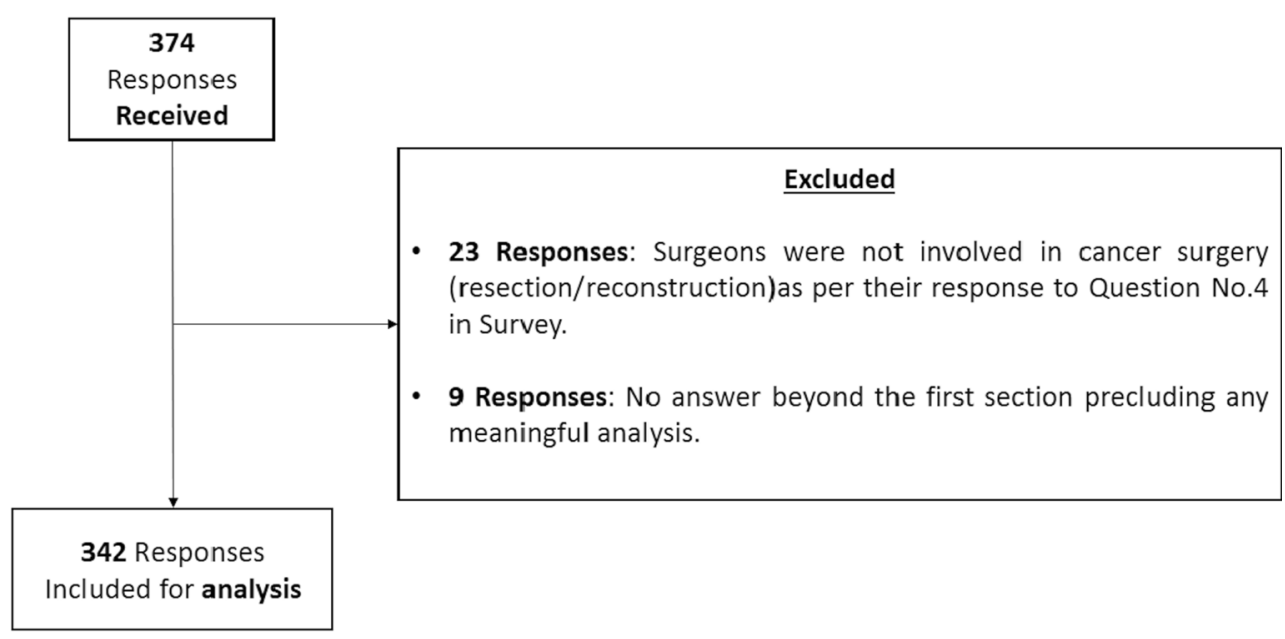

skin issues (dermatitis/acne), and headache. A large number of surgeons faced a combination of the above issues $(n=136$, $39.8 \%$ ), the most common combination being breathing difficulties/suffocation, headache, and communication difficulties $(n=36)$. Breathing difficulties and feelings of suffocation

Table 1 Basic demographic features of the respondents

\begin{tabular}{ll}
\hline Variables & Numbers $(\%)(n=342)$ \\
\hline Age group & \\
$25-30$ years & $28(8.2 \%)$ \\
$31-40$ years & $177(51.8 \%)$ \\
$41-50$ years & $91(26.6 \%)$ \\
$51-60$ years & $37(10.8 \%)$ \\
$>60$ years & $9(2.6 \%)$ \\
Gender & \\
Men & $275(80.4 \%)$ \\
Women & $67(19.6 \%)$ \\
Prior health issues (like headache and dermatitis) & \\
Yes & $39(11.4 \%)$ \\
No & $303(88.6 \%)$ \\
Specialty & \\
General surgical oncology & $95(27.8 \%)$ \\
Site-specific surgical oncology & $241(70.4 \%)$ \\
Plastic and reconstructive surgery & $6(1.8 \%)$ \\
Type of institute (presently working) & \\
Government & $155(45.3 \%)$ \\
Private & $176(51.5 \%)$ \\
NGO based & $11(3.2 \%)$ \\
Number of cases operated (since national lockdown) & $194(56.7 \%)$ \\
$<5$ cases/day & $104(30.4 \%)$ \\
$6-10$ cases/day & $23(6.7 \%)$ \\
$11-15$ cases/day & $5(1.5 \%)$ \\
$>20$ cases/day & $16(4.7 \%)$ \\
COVID-19 testing before routine cancer surgery & \\
Yes & $319(93.3 \%)$ \\
No & $23(6.7 \%)$ \\
\hline & \\
& \\
\hline &
\end{tabular}

were more with the use of N-95 masks (with or without the 3 -ply mask $(p<0.001)$, in comparison with the face respirators $(p<0.001)$. Surgeons who used face respirators had more problems with communication $(p=0.005)$. The eye protection components of PPE caused a variety of issues such as poor visibility, fogging, headache, discomfort, and inability to use loupes. A large number of surgeons faced a combination of the above issues $(n=199,58.2 \%)$, the most common combination being issues of poor visibility and fogging $(n=42)$. Both goggles and face shields were associated with poor visibility $(p<0.001)$ and fogging $(p=0.017)$ when compared to the use of routine prescription glasses or when none were used. The majority of the surgeons were not satisfied with the visibility that was available with the use of eye protection components $(n=157,45.9 \%)$. However, very few respondents felt $(n=23,6.8 \%)$ that there was an increase in the number of complications attributable to the use of PPE.

The components of the PPE used during surgery that contributed to discomfort the most were the N-95 mask and the face shield. Moderate to a great deal of fatigue $(n=236,69 \%)$ and stress $(n=181,52.9 \%)$ were reported by the large majority of the surgeons. Factors that maximally attributed to the fatigue with the use of N-95 masks were headache $\{p<$ $0.001[11.34(4.76-27.05)]\}$ followed by breathing difficulties/suffocations $\{\mathrm{p}<0.001$ [4.24 (2.49-7.47)]\} (Table 3). Similarly, factors that maximally attributed to the stress with the use of $\mathrm{N}-95$ masks were dryness of mouth $\{p<$ 0.001 [3.35(1.82-6.17)]\}, followed by fogging (due to eye protection device) $\{p<0.001$ [3.61(1.93-6.77)]\}, and breathing difficulty/suffocation $\{p=0.034[1.68(1.04-2.72)]\}$ (Table 3$)$. The majority of the surgeons $(n=223,65.2 \%)$ felt that they were unable to enjoy the surgeries compared to earlier due to routine use of PPE.

Physical exercise and frequent breaks between surgeries followed by meditation/yoga were the common measures taken by the respondents to minimize the stress and fatigue resulting from the use of PPE overall. Surgeons who reported 


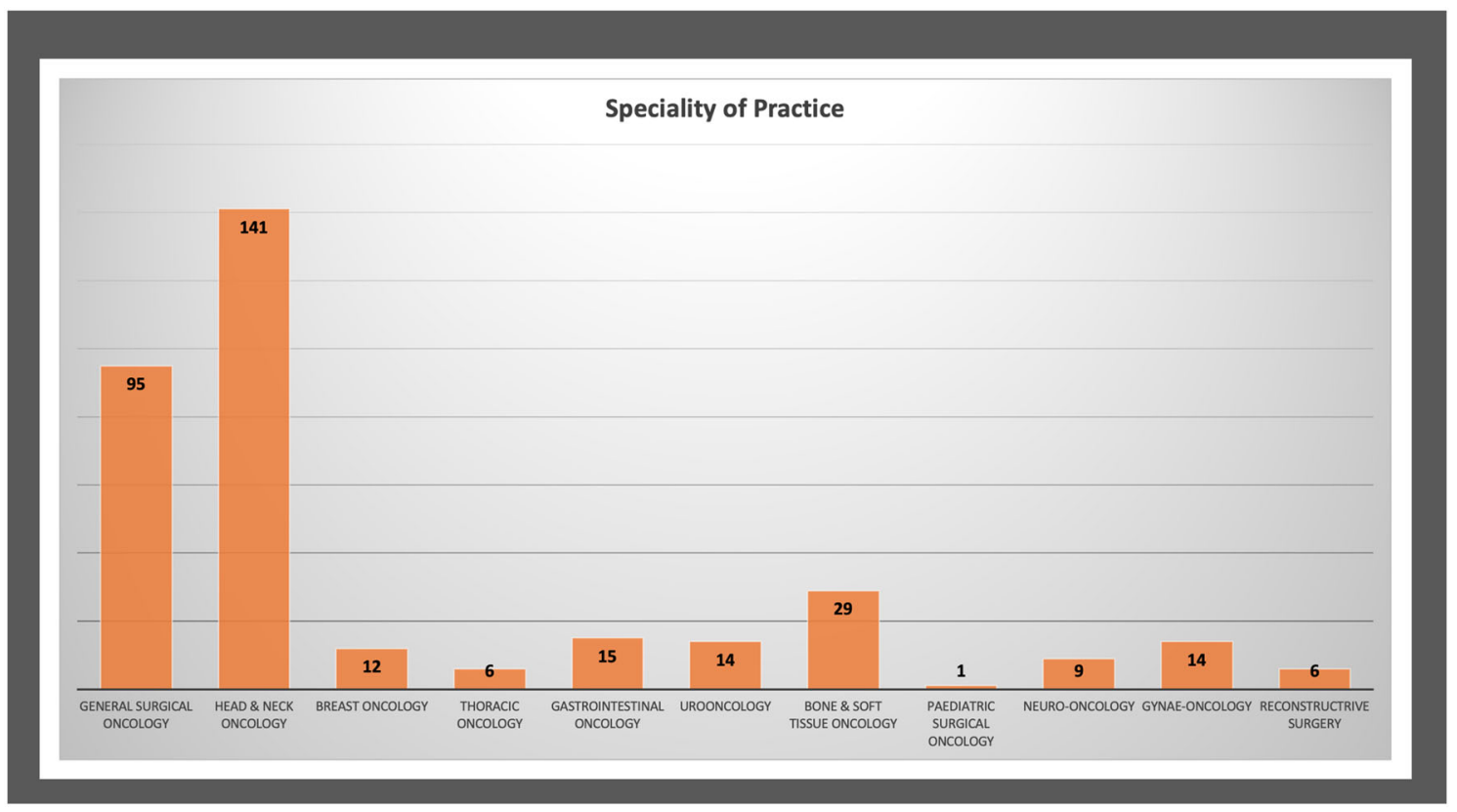

Fig. 2 Details of the practicing specialty of the respondents

moderate to high levels of stress and fatigue took frequent breaks between surgery and did yoga/meditation as a stress relief measure $(p=0.004)$.

\section{Discussion}

India currently stands second $(9,884,100$ cases) in the list of countries with confirmed COVID-19 cases (https://www.who. int/emergencies/diseases/novel-coronavirus-2019). As the country starts to open up after a lengthy period of lockdown (https://en.wikipedia.org/wiki/COVID-19_pandemic_ lockdown_in_India), patients diagnosed with cancer from across the country seeking treatment for their illness have begun accessing care in high-volume hospitals like ours. There is a resultant rise in the number of cancer surgeries being performed at these institutes. Our survey confirms that most centers in India perform RT-PCR tests for COVID-19 before elective cancer surgeries. However, the sensitivity of the RT-PCR employed for testing of COVID-19 infection is between 52 and $72 \%$ [11]. Hence, abundant precaution, by donning appropriate PPE, must be taken even if patients test negative for COVID-19 in a high-prevalence country like India.

Our nationwide survey showed that N-95masks/eye protection devices/double gloves/gowns (reusable/disposable) with or without shoe covers were the most often used combination of PPE used by cancer surgeons during the pandemic. Among these, the N-95 masks and the eye protection component contributed the most to surgeon discomfort. Four troublesome issues, i.e., headache, dryness of mouth, breathing difficulty/suffocation (attributable to the use of masks), and fogging (caused by the eye protection component), were the common reasons for increased stress and fatigue.

\section{Headache}

In our study, 43\% reported headaches with the use of PPE. Most respondents in our study attributed this to the use of N-95 masks (20\%) followed by both masks and eye protection devices (16\%). Only $7 \%$ attributed this to the use of eye protection devices alone. The possible reasons for this headache could be mechanical compression, hypoxemia, hypercarbia, and stress $[8,12-14]$. Pressure and/or tractional force from the mask and/ or the eye protection device can cause local tissue damage and irritation of the underlying (superficial) sensory nerve endings [15]. Also, the neck strain attributable to the use of these PPE components could stimulate headaches [16, 17]. Ong and colleagues in their cross-sectional study among $\mathrm{HCW}$ in high-risk areas of National University Hospital, Singapore, during the COVID-19 pandemic found that most of their respondents developed de novo headaches with PPE usage of $>4 \mathrm{~h}$ per day [6]. Most of the respondents agreed that these headaches influenced their work performance. Possible solutions suggested have been to wear the mask for a shorter duration, which may prevent or reduce the severity of the headaches [8]. This may translate into breaks between surgeries.

\section{Breathing Difficulty/Suffocation}

In our survey, a high percentage of respondents $(56 \%)$ had mentioned perceptions of breathing difficulty/suffocation. This was higher with those using N-95 masks and less among those using the face respirators. Though the N-95 mask offers 
Table 2 Details of PPE usage among the respondents and issues with its use
Variables

Number $(\%)(n=342)$

PPE used during routine cancer surgery in COVID negative patients

$\mathrm{N}-95+$ eye protection + single gloves + reusable gowns

$39(11.4 \%)$

$\mathrm{N}-95+$ eye protection + single gloves + disposable gowns + shoe covers

$31(9.1 \%)$

$\mathrm{N}-95+$ eye protection + double gloves + reusable gowns

$112(32.7 \%)$

$\mathrm{N}-95+$ eye protection + double gloves + disposable gowns + shoe covers

$160(46.8 \%)$

Agreement with present PPE use at their respective Institutes

Strongly agree

$115(33.6 \%)$

Agree

$165(48.2 \%)$

Neither agree nor disagree

$43(12.6 \%)$

Disagree

$18(5.3 \%)$

Strongly disagree

$1(0.3 \%)$

Type of mask used

$\mathrm{N}-95$ only

$75(21.9 \%)$

$\mathrm{N}-95+3$-ply

$115(33.6 \%)$

$20(5.8 \%)$

$54(15.8 \%)$

Face respirators

$9(2.6 \%)$

Only 3-ply

Combinations

$69(20.2 \%)$

Type of eye protection used

Face shield

$180(52.6 \%)$

Goggles

$102(29.8 \%)$

Both

$29(8.5 \%)$

Others

$14(4.1 \%)$

None

$17(5 \%)$

Use of eye protection

Always

$264(77.2 \%)$

Never

$17(5 \%)$

Only for aerosol generating procedures (AGP)

$61(17.8 \%)$

Surgical gloves

Always used double gloves

$201(58.8 \%)$

Always used single gloves

$88(25.7 \%)$

$53(15.5 \%)$

Double gloves after COVID-19 pandemic

$8(2.3 \%)$

Very comfortable

$8(2.3 \%)$

Neither comfortable nor uncomfortable

$31(9.1 \%)$

Uncomfortable but tolerable

$171(50 \%)$

Can be better

$24(7 \%)$

Very uncomfortable

$68(19.9 \%)$

No response

$40(11.7 \%)$

Necessity to take forced breaks in between surgery

Yes

$82(24 \%)$

No

$238(69.6 \%)$

No response

$22(6.4 \%)$

Surgery duration with PPE

$1 \mathrm{~h}$

$42(12.3 \%)$

$2 \mathrm{~h}$

$74(21.6 \%)$

$97(28.4 \%)$

$106(31 \%)$

$>3 \mathrm{~h}$

$23(6.7 \%)$ 
Table 2 (continued)

\begin{tabular}{l}
\hline Variables \\
\hline Issues with use of face asks including respirator \\
Combinations $\$$ \\
Communication \\
Dryness of mouth \\
Headache \\
Breathing issues/suffocations \\
Dehydration \\
Fogging \\
None \\
No response \\
Issues with use of eye protection \\
Combination* \\
Poor visibility/fogging \\
Discomfort \\
None \\
Others \\
No response \\
Visibility with eye protection \\
Very satisfied \\
Satisfied \\
Neither satisfied nor dissatisfied \\
Dissatisfied \\
Very dissatisfied \\
No response \\
Stess
\end{tabular}

Number $(\%)$

$(n=342)$

Issues with use of face asks including respirators

Combinations $^{\$}$

$136(39.8 \%)$

Communication

$41(12 \%)$

$4(1.2 \%)$

$78(22.8 \%)$

$40(11.7 \%)$

$1(0.3 \%)$

$4(1.2 \%)$

$16(4.7 \%)$

$22(6.4 \%)$

Issues with use of eye protection

$199(58.2 \%)$

$84(24.6 \%)$

$6(1.8 \%)$

$18(5.3 \%)$

$12(3.7 \%)$

$22(6.4 \%)$

Stress during surgery during COVID pandemic

A great deal

$8(2.3 \%)$

A lot

$68(19.9 \%)$

$76(25.4 \%)$

$117(34.2 \%)$

$40(11.7 \%)$

$33(6.4 \%)$

Moderate

$26(7.6 \%)$

$52(15.2 \%)$

A little

$103(30.1 \%)$

None

$93(27.2 \%)$

No response

$46(13.5 \%)$

Problems with using more than one glove on fine motor skills ability

$22(6.4 \%)$

Yes

$82(24 \%)$

No

$238(69.6 \%)$

No response

$22(6.4 \%)$

Increase in intraoperative complication rates attributable due to PPE usage.

Yes

$23(6.8 \%)$

No

$297(86.8 \%)$

No response

$22(6.4 \%)$

Fatigue after surgery

A great deal

$44(12.9 \%)$

A lot

$87(25.4 \%)$

Moderate

$105(30.7 \%)$

A little

$64(18.7 \%)$

None

$20(5.8 \%)$

No response

$22(6.4 \%)$

Inability to enjoy surgery during the pandemic

Strongly agree

$62(18.1 \%)$ 
Table 2 (continued)

\begin{tabular}{ll}
\hline Variables & $\begin{array}{l}\text { Number (\%) } \\
(n=342)\end{array}$ \\
\hline Agree & $153(44.7 \%)$ \\
Neither agree nor disagree & $58(17 \%)$ \\
Disagree & $39(11.4 \%)$ \\
Strongly disagree & $8(2.3 \%)$ \\
No response & $22(6.4 \%)$ \\
Change in choice of surgery & \\
Yes & $92(26.9 \%)$ \\
No & $227(66.4 \%)$ \\
No response & $23(6.7 \%)$ \\
Type of surgery performed during lockdown & \\
Less minimally invasive surgery & $109(31.9)$ \\
More minimally invasive surgery & $54(15.8 \%)$ \\
No change & $156(45.6 \%)$ \\
No response & $23(6.7)$ \\
Measures taken to reduce stress/discomfort & \\
Meditation/yoga & $61(17.8 \%)$ \\
Workouts (exercise) & $98(28.7 \%)$ \\
Frequent breaks between surgery & $92(26.9 \%)$ \\
Others & $69(20.2 \%)$ \\
No response & $22(6.4 \%)$ \\
\hline
\end{tabular}

$\$$, *more than one issues combined

better protection from respiratory infections [17], its use is associated with a feeling of discomfort, possibly because of a higher breathing resistance [18]. Studies have shown that prolonged use of N-95 masks is associated with higher nasal resistance and different recovery processes when compared to the use of surgical masks [19]. These factors could contribute to the perceptions of breathing difficulties/suffocation that our respondents had reported.

Table 3 Univariate and multivariate analysis

\begin{tabular}{|c|c|c|c|c|c|c|}
\hline \multirow[t]{2}{*}{ Contributing Factors } & \multicolumn{2}{|c|}{ Stress due to PPE use } & \multicolumn{2}{|c|}{ Fatigue due to PPE use } & \multicolumn{2}{|c|}{$\begin{array}{l}\text { Inability to enjoy surgery due to } \\
\text { PPE use }\end{array}$} \\
\hline & $\begin{array}{l}\text { Univariate } \\
\text { analysis } \\
\text { ( } p \text { value) }\end{array}$ & $\begin{array}{l}\text { Multivariate Analysis } \\
(p \text { value and OR with } \\
95 \% \mathrm{CI})\end{array}$ & $\begin{array}{l}\text { Univariate } \\
\text { analysis ( } p \\
\text { value) }\end{array}$ & $\begin{array}{l}\text { Multivariate analysis } \\
\text { ( } p \text { value and OR with } \\
95 \% \mathrm{CI})\end{array}$ & $\begin{array}{l}\text { Univariate } \\
\text { analysis } \\
\text { ( } p \text { value) }\end{array}$ & $\begin{array}{l}\text { Multivariate analysis } \\
(p \text { value and OR with } \\
95 \% \mathrm{CI})\end{array}$ \\
\hline \multicolumn{7}{|l|}{ Mask } \\
\hline Skin issues & 0.435 & - & 0.09 & - & 0.523 & - \\
\hline Difficulty in communication & 0.101 & - & 0.315 & - & 0.639 & - \\
\hline Dryness of mouth & $<0.001^{`}$ & $\begin{array}{l}<0.001 \\
\quad[3.35(1.82-6.17)]\end{array}$ & 0.02 & - & 0.016 & $\begin{array}{l}0.025 \\
\quad[4.12(1.19-14.25)]\end{array}$ \\
\hline $\begin{array}{l}\text { Breathing difficulty/feeling of } \\
\text { suffocation }\end{array}$ & 0.017 & $0.034[1.68(1.04-2.7)]$ & $<0.001$ & $<0.001[4.24(2.4-7.47)]$ & $<0.001$ & $\begin{array}{l}<0.001 \\
\quad[5.18(2.4-11.18)]\end{array}$ \\
\hline Headache & 0.004 & - & $<0.001$ & $\begin{array}{l}<0.001 \\
\quad[11.34(4.76-27.05)]\end{array}$ & 0.016 & - \\
\hline \multicolumn{7}{|l|}{ Eye protection component } \\
\hline Poor visibility & 0.028 & & 0.001 & - & 0.04 & - \\
\hline Fogging & $<0.001$ & $\begin{array}{l}<0.001 \\
\quad[3.61(1.93-6.77)]\end{array}$ & 0.139 & - & 0.174 & - \\
\hline Uncomforting & 0.674 & - & 0.05 & - & 0.06 & - \\
\hline Incompatible with loupes & 0.151 & - & 0.34 & - & 0.282 & - \\
\hline Headache & 0.319 & - & $<0.001$ & - & 0.334 & - \\
\hline
\end{tabular}




\section{Dryness of Mouth}

Of the respondents in our study, 22\% reported experiencing dryness of the mouth. With the use of $\mathrm{N}-95$ masks/respirators, there is a switch from nasal to oral breathing in most adults with higher energy expenditure [20]. The heat and amount of water lost via expired air are significantly greater with mouth breathing than nasal breathing, especially during high workload [21]. These factors could contribute to the dryness of mouth while using the N-95 masks/face respirators. Some of the strategies to minimize dryness of mouth include educating the user to breathe nasally, to use masks with exhalation valves and minimal dead space within [21]. The Centers for Disease Control and Prevention (CDC) had earlier warned against the use of masks with (one-way) exhalation valves or vents, as the exhaled air may spread respiratory droplets to others [22]. However, these masks/respirators can be used while wearing a face shield or, if tolerable, a disposable mask over the respirator/mask with valves to prevent the spread of respiratory droplets to others [23].

\section{Fogging}

Some studies, including our previous study, have reported impaired visibility due to fogging by the majority of their survey respondents $[9,10]$. In the present survey too, fogging was an important issue associated with the use of eye protection contributing to the overall discomfort related to the use of PPE. Fog-resistant eye protection may overcome some of these drawbacks. As an alternative, $\mathrm{Hu}$ and colleagues had suggested the use of either washing-up liquids or hand sanitizer on the protective eyewear to prevent fogging. The authors suggested it be evenly applied with gauze or cotton swabs and use subsequently after drying thoroughly [24].

\section{Stress Relief Measures}

Multiple factors have played a role in affecting the mental health of the surgeon during the COVID-19 pandemic. Some of them involve issues faced with the use of personal protective equipment. HCWs must be aware of the impact of the use of PPE on their well-being. Breaks in between surgeries may help minimize discomfort and fatigue. They must also hydrate themselves adequately to minimize dehydration (https://blogs.cdc.gov/niosh-science-blog/2020/06/10/ppeburden/). In our study, respondents used exercise (30.6\%), took frequent breaks between surgeries $(28.7 \%)$, and did yoga/meditation (19\%) to overcome the stress and discomfort. Other authors have suggested multiple coping mechanisms for surgeons that could be adopted during the pandemic [25].

The strength of our study is that it is among the few attempts made to assess surgeon discomfort associated with the use of PPE during routine cancer surgeries in the pandemic. It brings to the forefront the common issues about its use along with the measures that were taken by these surgeons to overcome them from a reasonable number of responses received. The limitation of the study, however, is that we could not go into the details of the coping measures adopted by the respondents to overcome their stress and fatigue.

\section{Conclusions}

In this national survey, among surgeons performing cancer surgery in COVID-19 negative patients, it was found that the N-95mask/eye protection devices/double gloves/reusable gowns were the most common combination of PPE used among the respondents. The N-95 mask and the eye protection device (face shield) were components of the PPE maximally contributing to discomfort and fatigue. Workouts, taking frequent breaks between surgery, and yoga/meditation were common measures taken by the respondents to overcome the stress and fatigue caused by the use of PPE. The findings from this study could help surgeons better understand the various issues associated with the use of PPE and help them adopt various strategies to minimize them.

Supplementary Information The online version contains supplementary material available at https://doi.org/10.1007/s13193-021-01316-6.

\section{Declarations}

Ethics Approval The authors confirm that all ethical standards were adhered to while doing this study. The study was approved by our institutional ethics committee. The study was also registered with the Clinical Trials Registry of India (CTRI/2020/08/027050).

Consent to Participate No identifying information about participants is available in the article. However, all participants have given consent for participation in the survey (incorporated in the survey questionnaire itself).

Conflict of Interest The authors declare no competing interests.

Meeting Presentations None

\section{References}

1. Forrester JD, Nassar AK, Maggio PM, Hawn MT (2020) Precautions for operating room team members during the COVID-19 pandemic. J Am Coll Surg 230(6):1098-1101

2. Moletta L, Pierobon ES, Capovilla G et al (2020) International guidelines and recommendations for surgery during Covid-19 pandemic: a systematic review. Int J Surg 79:180-188 [published online ahead of print, 2020 May 23] 
3. Kucirka LM, Lauer SA, Laeyendecker O, Boon D, Lessler J (2020) Variation in false-negative rate of reverse transcriptase polymerase chain reaction-based SARS-CoV-2 tests by time since exposure. Ann Intern Med

4. Lau JT, Fung KS, Wong TW et al (2004) SARS transmission among hospital workers in Hong Kong. Emerg Infect Dis 10: 280-286

5. Seto WH, Tsang D, Yung RW et al (2003) Effectiveness of precautions against droplets and contact in prevention of nosocomial transmission of severe acute respiratory syndrome (SARS). Lancet 361:1519-1520

6. Ong JJ, Bharatendu C, Goh Y et al (2020) Headaches associated with personal protective equipment - a cross-sectional study among frontline healthcare workers during COVID-19. Headache 60:864877

7. Gheisari M, Araghi F, Moravvej H, Tabary M, Dadkhahfar S (2020) Skin reactions to non-glove personal protective equipment: an emerging issue in the COVID-19 pandemic. J Eur Acad Dermatol Venereol 34:297-298

8. Lim EC, Seet RC, Lee KH, Wilder-Smith EP, Chuah BY, Ong BK (2006) Headaches and the N95 face-mask amongst healthcare providers. Acta Neurol Scand 113(3):199-202

9. Prakash G, Shetty P, Thiagarajan S et al (2020) Compliance and perception about personal protective equipment among health care workers involved in the surgery of COVID-19 negative cancer patients during the pandemic. J Surg Oncol. https://doi.org/10. 1002/jso. 26151

10. Yánez Benítez C, Güemes A, Aranda J et al (2020) International cooperation group on PPE and emergency surgery, Ramos JP, Rangelova E, Muñoz M, Yánez C Sr. impact of personal protective equipment on surgical performance during the COVID-19 pandemic. World J Surg 20:1-6

11. Wang W, Xu Y, Gao R, Lu R, Han K, Wu G, Tan W (2020) Detection of SARS-CoV-2 in different types of clinical specimens. JAMA. https://doi.org/10.1001/jama.2020.3786

12. Rebmann T, Carrico R, Wang J (2013) Physiologic and other effects and compliance with long-term respirator use among medical intensive care unit nurses. Am J Infect Control 41:1218-1223

13. Radonovich LJ Jr, Cheng J, Shenal BV et al (2009) Respirator tolerance in health care workers. JAMA 301:36-38

14. Shenal BV, Radonovich LJ Jr, Cheng J, Hodgson M, Bender BS (2012) Discomfort and exertion associated with prolonged wear of respiratory protection in a health care setting. J Occup Environ Hyg 9:59-64

15. Krymchantowski AV (2010) Headaches due to external compression. Curr Pain Headache Rep 14:321-324

16. Liang Z, Galea O, Thomas L, Jull G, Treleaven J (2019) Cervical musculoskeletal impairments in migraine and tension type headache: a systematic review and meta-analysis. Musculoskelet Sci Pract 42:67-83

17. Bałazy A, Toivola M, Adhikari A, Sivasubramani SK, Reponen T, Grinshpun SA (2006) Do N95 respirators provide 95\% protection level against airborne viruses, and how adequate are surgical masks? Am J Infect Control 34(2):51-57

18. Lee HP, de Wang Y (2011) Objective assessment of increase in breathing resistance of $\mathrm{N} 95$ respirators on human subjects. Ann Occup Hyg 55(8):917-921

19. Li Y, Tokura H, Guo YP, Wong ASW, Wong T, Chung J, Newton E (2005) Effects of wearing N95 and surgical facemasks on heart rate, thermal stress and subjective sensations. Int Arch Occup Environ Health 78(6):501-509

20. Harber P, Beck J, Luo J (1997) Study of respirator effect on nasaloral flow partition. Am J Ind Med 32:408-412

21. Roberge RJ, Kim JH, Coca A (2012) Protective facemask impact on human thermoregulation: an overview. Ann Occup Hyg 56(1): $102-112$

22. Thebault R, Fitz A. Face masks with valves or vents do not prevent spread of the coronavirus, CDC says. Available at: https://www. washingtonpost.com/health/2020/08/13/cdc-mask-guidancemasks-valves/. Accessed August 8, 2020

23. Chang JC, Johnson JS, Olmsted RN (2020) Demystifying theoretical concerns involving respirators with exhalation valves during COVID-19 pandemic [published online ahead of print, 2020 Aug 26]. Am J Infect Control S0196-6553(20):30815-30814. https://doi.org/10.1016/j.ajic.2020.08.031

24. (2020) Prevention of fogging of protective eyewear for medical staff during the COVID-19 pandemic. J Emerg Nurs 46(5):564 566. https://doi.org/10.1016/j.jen.2020.05.003

25. Balasubramanian A, Paleri V, Bennett R, Paleri V (2020) Impact of COVID-19 on the mental health of surgeons and coping strategies. Head Neck 42(7):1638-1644. https://doi.org/10.1002/hed.26291

Publisher's Note Springer Nature remains neutral with regard to jurisdictional claims in published maps and institutional affiliations. 